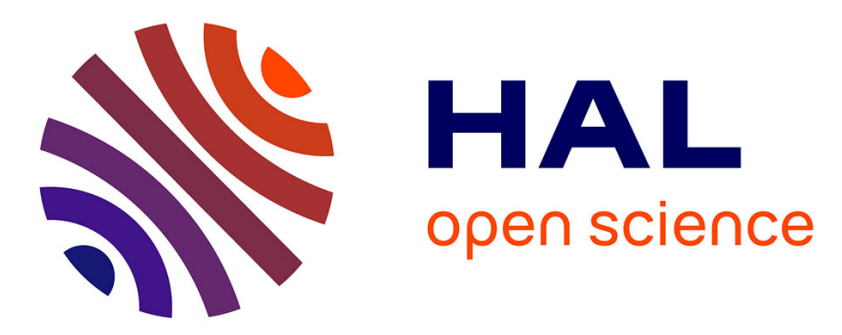

\title{
Microelasticity model of random alloys. Part I: mean square displacements and stresses
}

Pierre-Antoine Geslin, David Rodney

\section{To cite this version:}

Pierre-Antoine Geslin, David Rodney. Microelasticity model of random alloys. Part I: mean square displacements and stresses. Journal of the Mechanics and Physics of Solids, 2021, 153, pp.104479. 10.1016/j.jmps.2021.104479 . hal-03223375

\section{HAL Id: hal-03223375 \\ https://hal.science/hal-03223375}

Submitted on 10 May 2021

HAL is a multi-disciplinary open access archive for the deposit and dissemination of scientific research documents, whether they are published or not. The documents may come from teaching and research institutions in France or abroad, or from public or private research centers.
L'archive ouverte pluridisciplinaire HAL, est destinée au dépôt et à la diffusion de documents scientifiques de niveau recherche, publiés ou non, émanant des établissements d'enseignement et de recherche français ou étrangers, des laboratoires publics ou privés. 


\title{
Microelasticity model of random alloys. Part I: mean square displacements and stresses
}

\author{
Pierre-Antoine Geslin \\ Univ. Lyon, CNRS, INSA Lyon, UCBL, MATEIS, UMR5510, 69621 Villeurbanne, \\ France \\ E-mail: pierre-antoine.geslin@insa-lyon.fr
}

\begin{abstract}
David Rodney
Univ. Lyon, Université Claude Bernard Lyon 1, CNRS, Institut Lumière Matière, LYON, F-69622 France
\end{abstract}

E-mail: david.rodney@univ-lyon1.fr

\begin{abstract}
In concentrated solid solutions, the random distribution of elements of different sizes induces characteristic displacement and stress fields at the root of solid solution strengthening. The aim of this two-part article is to derive the statistical properties of these elastic fields. The present Part I focuses on the variance of the elastic fields, while Part II addresses their spatial correlations. In this first part, we develop two elastic models of random solid solutions, based respectively on realspace and spectral methods, to derive the mean square displacement and shear stress. Both approaches hold advantages and drawbacks that are discussed here. We show in particular that both the mean square displacement and shear stress are directly proportional to the variance of the atomic eigenstrains, which embodies the atomic size differences and simplifies to the classical lattice mismatch parameter if the alloy satisfies Vegard's law. This allows to clarify the scaling relations between various quantities (mismatch parameter, mean square displacement and yield stress) and to bridge energy-based and stress-based models of concentrated solid solution strengthening proposed in the literature. The elastic predictions for the mean-square displacement and stresses are also successfully compared with atomistic results obtained for a model Lennard-Jones system and with a more complex Al-Mg interatomic potential.
\end{abstract}

The physical mechanism underlying solid solution strengthening is that solute atoms introduce a disturbance in the perfect lattice that pins dislocations, therefore increasing the yield stress of the alloy. This property is largely taken advantage of in the latest generations of technical alloys that contain more alloying elements in larger quantities. High entropy alloys (HEA) represent an extreme case of this trend, with five or more elements mixed in comparable quantities in a disordered solid solution. These novel alloys have attracted extreme attention in the past decade because of their outstanding mechanical properties, including both a high yield stress and a large toughness [1, 2]. Solid solution strengthening has been recognized as a major strengthening mechanism 
in these alloys $[1,3,4,5]$, but predicting and optimizing this effect for concentrated alloys and HEA appears as a formidable task considering the complexity of the alloys and the wide composition range to explore [6].

The seminal works of Fleisher [7] and Labusch [8, 9] investigated solute strengthening from the viewpoint of the interaction between solute atoms and the pressure field of a dislocation. The magnitude of the strengthening was then related to the size difference between the solute atoms and the solvent. In the case of concentrated alloys, the distinction between solute and solvent breaks down and the size difference of the alloy elements is better measured with respect to the average atomic radius. A parameter widely accepted to quantify the size difference between the alloy components is the so-called atomic size mismatch parameter $[2,10,11,12]$ :

$$
\delta=\sqrt{\sum_{i} c_{i}\left(\frac{r_{i}}{\bar{r}}-1\right)^{2}} \quad \text { with } \quad \bar{r}=\sum_{i} c_{i} r_{i}
$$

where $r_{i}$ and $c_{i}$ are respectively the atomic radius and concentration of each element and $\bar{r}$ is the average radius. The atomic radius is somewhat ill-defined since the same element can adopt different radii in different chemical environments $[13,14]$. When the pure elements share the same crystalline structure, the corresponding lattice spacing can be used in Eq. (1) in lieu of the atomic size [15]. Based on experimental observations, it has been suggested that the mechanical properties of the alloy such as the yield stress [2] or the hardness [16] correlate with the mismatch parameter $\delta$, which has been used as an empirical parameter to guide the design of HEA [15].

To better quantify the role of the atomic size difference on the solid solution strengthening in face-centered cubic random alloys, Leyson et al. [9] and Varvenne et al. [17] extended Labusch's approach and related the yield stress of an alloy to the misfit volumes of its components (and therefore to the $\delta$ parameter). This approach relies on the evaluation of the interaction energy between dislocations and solutes of different sizes. The model can be parameterized from DFT calculations but in its simplest elastic version, it relates the yield stress at $0 \mathrm{~K}$ denoted $\tau_{y 0}$ to the misfit parameter $\delta$ as $[17,18,19]$ :

$$
\tau_{y 0} \sim\left(\frac{1}{\Gamma}\right)^{1 / 3}\left(\mu \frac{1+\nu}{1-\nu}\right)^{4 / 3} \delta^{4 / 3}
$$

where $\Gamma$ is the line tension of the dislocation and $\mu$ and $\nu$ are the isotropic elastic constants of the random alloy. We note that Varvenne's approach is not limited to $0 \mathrm{~K}$ but also incorporates a temperature dependence by considering a characteristic energy barrier to overcome for dislocation glide. While the role of temperature is of paramount importance for applications, it will not be discussed in the present paper. This theory was found to match experimental measurements for several families of alloys $[17,19,20,21,22,23,24]$.

Another consequence of the atomic size differences are the displacements of the atoms from their lattice sites, that some authors refer to as "lattice distortions" [1, 2]. 
These atomic displacements can be quantified by the mean square atomic displacement, $\left\langle u_{a t}^{2}\right\rangle$, equal to the variance of the displacements when the average alloy is used as reference (i.e. such that $\left\langle u_{a t}\right\rangle=0$ ). Mean square displacements have been measured experimentally by X-ray diffraction [1, 25, 26, 27, 28], high resolution transmission electron microscopy [26] as well as neutron diffraction [29, 30]. They have also been computed in atomistic models of solid solutions based on the density function theory (DFT) $[13,14,31,32]$. Moreover, to explain results obtained from X-ray diffraction experiments, it has been proposed that the mean square displacement should be proportional to the square of the size mismatch parameter, such that $[25,26]$ :

$$
\left\langle u_{a t}^{2}\right\rangle \sim \sum_{i} c_{i}\left(r_{i}-\bar{r}\right)^{2} \sim \delta^{2}
$$

Such a linear scaling has been reported in atomistic models [13], although with deviations which depend on the way atomic sizes were defined. Moreover, a linear scaling was empirically evidenced between the yield stress of a HEA measured experimentally and extrapolated to $0 \mathrm{~K}$ and the root mean square displacement $\sqrt{\left\langle u_{a t}^{2}\right\rangle}$ computed from DFT calculations [31]. Combining this empirical scaling with Eq. (2), Nöhring and Curtin [18] showed on atomic models of HEA an approximate scaling between the mean square atomic displacement and the $\delta$-parameter, $\left\langle u_{a t}^{2}\right\rangle \sim \delta^{8 / 3}$, in contradiction with Eq. (3).

Atomic size differences also lead to internal stresses that have not been extensively studied in random alloys, probably because they are difficult to measure experimentally. However, another viewpoint of the study of solute/dislocation interactions consists in considering the dislocation evolving in the internal stress field emerging from the solid solution. Using this viewpoint, one can rely on the large body of literature devoted to the depinning transition of elastic lines in random media $[33,34,35,36]$. In the case of a random uncorrelated stress environment, basic scaling arguments enable to estimate the critical resolved shear stress at $0 \mathrm{~K}$ as a function of the amplitude of the random stress field:

$$
\tau_{y 0} \sim \frac{\left\langle\tau^{2}\right\rangle^{2 / 3}}{\Gamma^{1 / 3}}
$$

where $\left\langle\tau^{2}\right\rangle$ denotes the variance of the internal shear stress field acting on the dislocation and emerging from the random alloy environment and $\Gamma$ is again the dislocation line tension. This approach relies on the knowledge of internal stress fields acting on the dislocation rather than on the dislocation/solutes interaction energies as in Labuschtype approaches $[8,9,17]$. We note that the scaling in Eq. (4) was obtained assuming an uncorrelated noise environment, while we know that internal stresses can display spatial correlations emerging from elasticity [37]. It appears therefore as an important task to characterize the statistical properties of not only the displacements, but also of the internal stresses emerging from a random solid solution.

In this two-part article, we develop an elastic model of random alloys and focus on the statistical properties (variance and spatial correlations) of both the displacement and stress fields. The present Part I of this work is dedicated to the mean square 
Microelasticity model of random alloys. Part I: mean square displacements and stresses4

displacements and stresses and their dependence on the alloy composition and elastic properties while Part II focuses on spatial correlations.

In this part I, we propose two different methods to compute the variance of the displacements and stresses. As described in section 1, both approaches rely on a description of the random alloy as an elastic medium where atoms of different nature are modeled as dilatational inclusions with a site occupancy probability. The first method presented in section 2 is based on a real space approach first used by Nöhring and Curtin [18], where the displacement (and stress) at a given point is expressed as the sum of the displacements (and stresses) due to all surrounding atoms. This real-space method is however not practical to study spatial correlations. We therefore introduce in section 3, a spectral method based on the microelasticity theory [38, 39], which shares similarities with models used to predict scattering from random solid solutions in diffraction experiments $[40,41,42]$. In section 4, our analytical predictions are compared to atomistic results performed in a model Lennard-Jones system and in more realistic $\mathrm{Al}-\mathrm{Mg}$ alloys whose isotropic nature fits well with the assumptions of the elastic model.

\section{Elastic framework of random alloys}

In this study, we consider that a random alloy can be modeled as an elastic medium where atoms of different nature are described as dilatational inclusions with a given site occupancy probability.

\subsection{Description of the random alloy}

We consider a substitutional alloy described as an homogeneous elastic medium of elastic constants $C_{i j k l}$ in the anisotropic case that reduce to a shear modulus $\mu$ and a Poisson ratio $\nu$ for isotropic elasticity. The alloy is constituted of $N_{\text {elem }}$ elements of different nature. The atoms are modeled as elastic inclusions distributed randomly on a crystalline lattice as schematically represented in Fig. 1. Their eigenstrain is assumed diagonal and expressed as $\bar{\varepsilon}_{\alpha}^{00}=\varepsilon_{\alpha} \operatorname{diag}(1,1,1)$. In section 2 , the inclusions is assumed spherical and characterized by a constant eigenstrain, while in section 3 , the eigenstrain are spatially smeared with a Gaussian spreading function of width $a$ (see Fig. 1). We note $N$ the total number of atoms/inclusions placed on a lattice of volume $V=L_{x} L_{y} L_{z}$. The atomic volume is $v_{a t}=V / N$.

Every element is associated with a site occupancy random variable given by a Bernoulli distribution independent of the lattice site $[17,18]$. Thus, we do not consider any ordering of the species that may appear in real alloys [22, 43]. The probability of site $n$ to host an atom of type $\alpha$ is expressed as:

$$
P_{\alpha}(n)= \begin{cases}0 & \text { with probability } 1-c_{\alpha} \\ 1 & \text { with probability } c_{\alpha}\end{cases}
$$


Microelasticity model of random alloys. Part I: mean square displacements and stresses5

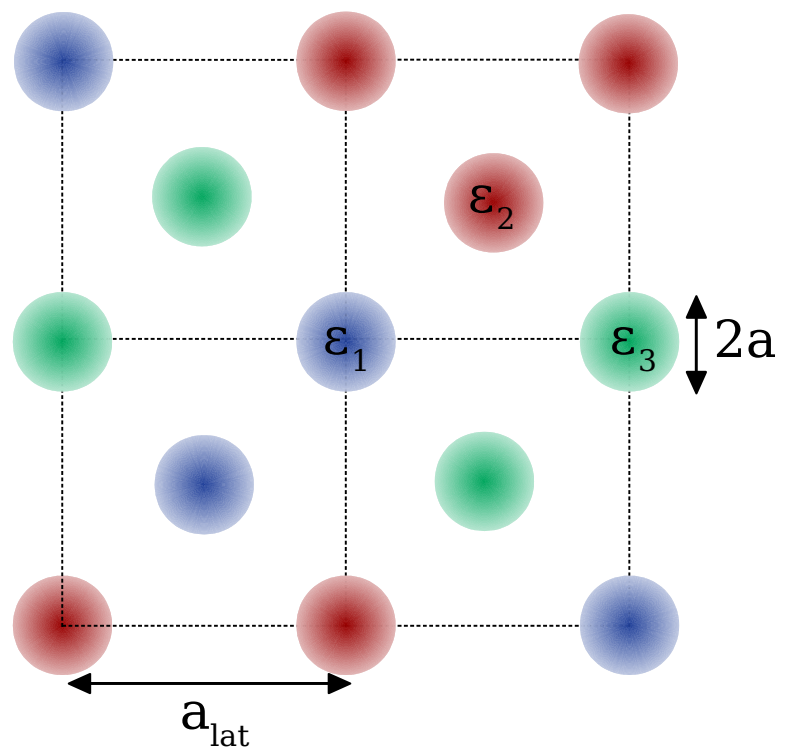

Figure 1. Distribution of eigenstrains on an atomic lattice. An eigenstrain $\varepsilon_{\alpha}$ is associated to each element $\alpha$. The Gaussian spreading of width $a$ used in section 3 is schematically represented here.

where $c_{\alpha}$ is the atomic concentration of element $\alpha$ in the random alloy. The covariance of the site occupancy variables is given by:

$$
\left\langle P_{\alpha}(n) P_{\beta}(m)\right\rangle=\left\{\begin{array}{ll}
\left\langle P_{\alpha}^{2}\right\rangle=c_{\alpha} & \text { if } \alpha=\beta \text { and } n=m \\
\left\langle P_{\alpha}\right\rangle^{2}=c_{\alpha}^{2} & \text { if } \alpha=\beta \text { and } n \neq m \\
0 & \text { if } \alpha \neq \beta \text { and } n=m \\
\left\langle P_{\alpha}\right\rangle\left\langle P_{\beta}\right\rangle=c_{\alpha} c_{\beta} & \text { if } \alpha \neq \beta \text { and } n \neq \mathrm{m}
\end{array},\right.
$$

where $\langle$.$\rangle denotes a statistical average. The third equality derives from the condition$ that there is a single atom per site, such that $P_{\alpha}(n)$ and $P_{\beta}(n)$ cannot be simultaneous non-zero if $\alpha \neq \beta$.

\subsection{Reference lattice and eigenstrains}

The eigenstrains $\varepsilon_{\alpha}$ describing the sizes of different atoms are defined with respect to a reference lattice for which several choices are possible. In a dilute alloy, a natural choice is to consider the solvent lattice as the reference. However, for concentrated solid solutions such as an HEA, a more convenient frame of reference is the lattice of the average alloy. This concept of average alloy was first introduced by Varvenne et al. [17] and later extended to atomistic modeling in Ref. [44]. The composition of the random alloy is denoted $\left(c_{1}, \ldots, c_{N_{\text {elem }}}\right)$ and the lattice spacing of the random alloy is $a_{\text {lat }}$. Following Ref. [17], we define the eigenstrain $\varepsilon_{\alpha}$ as the change of lattice spacing with composition around the alloy composition: 
Microelasticity model of random alloys. Part I: mean square displacements and stresses6

$$
\varepsilon_{\alpha}=\sum_{\beta=1}^{N_{\text {elem }}} \frac{c_{\beta}}{a_{l a t}}\left(\left.\frac{\partial a_{\text {lat }}}{\partial c_{\alpha}}\right|_{\bar{c}}-\left.\frac{\partial a_{\text {lat }}}{\partial c_{\beta}}\right|_{\bar{c}}\right) .
$$

With this definition, the eigenstrains have a zero mean:

$$
\sum_{\alpha=1}^{N_{\text {elem }}} c_{\alpha} \varepsilon_{\alpha}=0
$$

We note that the misfit volume $\Delta V_{\alpha}$ commonly used in the literature $[17,19,20$, $21,22,23,24]$ is directly proportional to $\varepsilon_{\alpha}$ :

$$
\Delta V_{\alpha}=3 v_{a t} \varepsilon_{\alpha}
$$

where $v_{a t}$ is the average atomic volume of the random alloy.

\section{Real space method}

Within the elastic framework described above, the real-space method detailed here evaluates the displacement (and stress) of a given atom as the sum of displacements (and stresses) emerging from all surrounding atoms described as elastic inclusions. This approach yields compact expressions for the mean square displacement (section 2.1) and stress (section 2.2).

\subsection{Mean square displacement}

We first focus on displacements following the approach described by Nöhring and Curtin [18]. The displacement field around an atom modeled as a spherical Eshelby inclusion of volume $v_{a t}$ and eigenstrain $\varepsilon$ is $[45,46] \ddagger$ :

$$
\boldsymbol{u}^{\text {Eshelby }}(\boldsymbol{r})=\varepsilon \mathcal{F}(\boldsymbol{r})=\varepsilon \frac{v_{a t}}{4 \pi} \frac{1+\nu}{1-\nu} \frac{\boldsymbol{r}}{|\boldsymbol{r}|^{3}}
$$

Reciprocally, the displacement of an atom located arbitrarily at the origin is given by the sum of all the displacements originating from all other atoms in the system:

$$
\boldsymbol{u}(\mathbf{0})=-\sum_{n=1}^{N} \varepsilon(n) \mathcal{F}\left(\boldsymbol{R}_{\boldsymbol{n}}\right)=-\sum_{n=1}^{N} \sum_{\alpha=1}^{N_{\text {elem }}} \varepsilon_{\alpha} P_{\alpha}(n) \mathcal{F}\left(\boldsymbol{R}_{\boldsymbol{n}}\right)
$$

where $\left\{\boldsymbol{R}_{\boldsymbol{n}}\right\}_{n=1, N}$ are the atomic/inclusion positions (with $\boldsymbol{R}_{\boldsymbol{n}}=0$ excluded). The second equality stems from the fact that $\varepsilon(n)=\sum_{\alpha}^{N_{\text {elem }}} \varepsilon_{\alpha} P_{\alpha}(n)$.

The displacement $\boldsymbol{u}(\mathbf{0})$ is therefore a random vector whose components $u_{i}(\mathbf{0})$ are expressed as sums of random variables $u_{i}(\mathbf{0})=\sum_{n=1}^{N} \eta_{n}=-\sum_{n=1}^{N} \sum_{\alpha} \varepsilon_{\alpha} P_{\alpha}(n) \mathcal{F}_{i}\left(\boldsymbol{R}_{\boldsymbol{n}}\right)$. $\ddagger$ the vectors such as $\boldsymbol{r}$ are denoted in bold font and $|\boldsymbol{r}|$ denotes norm of vector $\boldsymbol{r}$. 
Microelasticity model of random alloys. Part I: mean square displacements and stresses7

These variables are drawn from different distributions (depending on the distance $\left|R_{n}\right|$ ), which we checked satisfy the conditions of Lyapunov's central-limit theorem [47] as long as the inclusions are distributed homogeneously in space, which is obviously the case for a crystalline lattice. The displacement $\boldsymbol{u}(\mathbf{0})$ is therefore a random vector following a 3-dimensional normal distribution.

The atomic mean square displacement (MSD) denoted $\left\langle u_{a t}^{2}\right\rangle$ is then obtained by computing the variance of the displacements expressed in Eq. (11). Averaging over realizations, we have:

$$
\left\langle u_{\text {at }}^{2}\right\rangle=\left\langle\sum_{n=1}^{N} \sum_{m=1}^{N} \sum_{\alpha=1}^{N_{\text {elem }}} \sum_{\beta=1}^{N_{\text {elem }}} \varepsilon_{\alpha} \varepsilon_{\beta} P_{\alpha}(n) P_{\beta}(m) \mathcal{F}\left(\boldsymbol{R}_{\boldsymbol{n}}\right) \cdot \mathcal{F}\left(\boldsymbol{R}_{\boldsymbol{m}}\right)\right\rangle
$$

Following Ref. [17, 18], this sum can be decomposed as:

$$
\begin{aligned}
\left\langle u_{\text {at }}^{2}\right\rangle & =S_{1}+S_{2}+S_{3} \\
& =\left\langle\sum_{n=1}^{N} \sum_{\alpha=1}^{N_{\text {elem }}} \varepsilon_{\alpha}^{2} P_{\alpha}(n)^{2}\left|\mathcal{F}\left(\boldsymbol{R}_{\boldsymbol{n}}\right)\right|^{2}\right\rangle \\
& +\left\langle\sum_{n=1}^{N} \sum_{\alpha=1}^{N_{\text {elem }}} \sum_{\beta \neq \alpha}^{N_{\text {elem }}} \varepsilon_{\alpha} \varepsilon_{\beta} P_{\alpha}(n) P_{\beta}(n)\left|\mathcal{F}\left(\boldsymbol{R}_{\boldsymbol{n}}\right)\right|^{2}\right\rangle \\
& +\left\langle\sum_{n=1}^{N} \sum_{m \neq n}^{N} \sum_{\alpha=1}^{N_{\text {elem }}} \sum_{\beta=1}^{N_{\text {elem }}} \varepsilon_{\alpha} \varepsilon_{\beta} P_{\alpha}(n) P_{\beta}(m) \mathcal{F}\left(\boldsymbol{R}_{\boldsymbol{n}}\right) \cdot \mathcal{F}\left(\boldsymbol{R}_{\boldsymbol{m}}\right)\right\rangle
\end{aligned}
$$

Since $\left\langle P_{\alpha}(n)^{2}\right\rangle=c_{\alpha}$, we have:

$$
S_{1}=\sum_{\alpha=1}^{N_{\text {elem }}} c_{\alpha}^{2} \varepsilon_{\alpha}^{2} \sum_{n=1}^{N}\left|\mathcal{F}\left(\boldsymbol{R}_{\boldsymbol{n}}\right)\right|^{2}
$$

Also, $S_{2}=0$ because $\left\langle P_{\alpha}(n) P_{\beta}(n)\right\rangle=0$ if $\alpha \neq \beta$. Finally, since $\left\langle P_{\alpha}(n) P_{\beta}(m)\right\rangle=c_{\alpha} c_{\beta}$ when $m \neq n, S_{3}$ is rewritten as:

$$
S_{3}=\sum_{\alpha=1}^{N_{\text {elem }}} c_{\alpha} \varepsilon_{\alpha} \sum_{\beta=1}^{N_{\text {elem }}} c_{\beta} \varepsilon_{\beta} \sum_{n=1}^{N} \sum_{m \neq n}^{N} \mathcal{F}\left(\boldsymbol{R}_{\boldsymbol{n}}\right) \cdot \mathcal{F}\left(\boldsymbol{R}_{\boldsymbol{m}}\right)=0
$$

due to the average lattice condition in Eq. (8). Using the expression of $\mathcal{F}\left(\boldsymbol{R}_{\boldsymbol{n}}\right)$ in Eq. (10), we have:

$$
\left\langle u_{a t}^{2}\right\rangle=\frac{v_{a t}^{2}}{16 \pi^{2}}\left(\frac{1+\nu}{1-\nu}\right)^{2} \Delta \varepsilon^{2} \sum_{n=1}^{N} \frac{1}{\left|\boldsymbol{R}_{\boldsymbol{n}}\right|^{4}},
$$

where $\Delta \varepsilon^{2}$ is the variance of the eigentrains defined as:

$$
\Delta \varepsilon^{2}=\sum_{\alpha=1}^{N_{\text {elem }}} c_{\alpha} \varepsilon_{\alpha}^{2}-\left(\sum_{\alpha=1}^{N_{\text {elem }}} c_{\alpha} \varepsilon_{\alpha}\right)^{2}=\sum_{\alpha=1}^{N_{\text {elem }}} c_{\alpha} \varepsilon_{\alpha}^{2}
$$


Microelasticity model of random alloys. Part I: mean square displacements and stresses8

the second equality stemming from the use of the average alloy as reference frame. The sum over all lattice sites can be computed numerically in the limit of an infinite system. In the case of interest in section 4 of a FCC lattice with a lattice spacing $a_{l a t}$, we have:

$$
\sum_{F C C} \frac{1}{\left|\boldsymbol{R}_{\boldsymbol{n}}\right|^{4}} \simeq \frac{101.3}{a_{\text {lat }}^{4}} \simeq \frac{25.3}{v_{\text {at }} a_{\text {lat }}}
$$

where $v_{a t}=a_{\text {lat }}^{3} / 4$ is the atomic volume. Finally, we obtain:

$$
\left\langle u_{a t}^{2}\right\rangle \simeq \frac{25.3}{16 \pi^{2}} \frac{v_{a t} \Delta \varepsilon^{2}}{a_{\text {lat }}}\left(\frac{1+\nu}{1-\nu}\right)^{2} .
$$

\subsection{Mean square shear stress}

We now apply the same reasoning to the atomic stresses to obtain the atomic mean square shear stress (MSSS). We focus on the shear stress components that produce a Peach-Koehler force [48] on dislocations and control the yield stress of the alloy through Eq. (4). The shear stress around a spherical inclusion of volume $v_{a t}$ and dilatational eigenstrain $\varepsilon$ is given by $[39,45]$ :

$$
\tau_{i j}^{\text {Eshelby }}(\boldsymbol{r})=\varepsilon \mathcal{G}_{i j}(\boldsymbol{r})=-\varepsilon \frac{3 v_{a t} \mu}{2 \pi} \frac{1+\nu}{1-\nu} \frac{r_{i} r_{j}}{\boldsymbol{r}^{5}},
$$

and the shear stress at an atom located at the origin is:

$$
\tau_{i j}(\mathbf{0})=-\sum_{n=1}^{N} \varepsilon(n) \mathcal{G}_{i j}\left(\boldsymbol{R}_{\boldsymbol{n}}\right) .
$$

As for the displacements, Lyapunov's central-limit theorem [47] can be used to show that

this quantity follows a Gaussian distribution. Following the same averaging procedure as in section 2.1, we find:

$$
\left\langle\tau_{i j}^{2}\right\rangle=\frac{9 v_{a t}^{2} \mu^{2}}{4 \pi^{2}}\left(\frac{1+\nu}{1-\nu}\right)^{2} \Delta \varepsilon^{2} \sum_{n=1}^{N} \frac{R_{n, i}^{2} R_{n, j}^{2}}{\left|\boldsymbol{R}_{\boldsymbol{n}}\right|^{10}} .
$$

For a FCC lattice of lattice spacing $a_{\text {lat }}$, we have:

$$
\sum_{F C C} \frac{R_{n, i}^{2} R_{n, j}^{2}}{\left|\boldsymbol{R}_{\boldsymbol{n}}\right|^{10}} \simeq \frac{9.0175}{a_{\text {lat }}^{6}},
$$

yielding the following expression for the MSSS:

$$
\left\langle\tau_{a t}^{2}\right\rangle \simeq \frac{81.16}{16 \pi^{2}} \frac{v_{a t} \mu^{2} \Delta \varepsilon^{2}}{a_{\text {lat }}^{3}}\left(\frac{1+\nu}{1-\nu}\right)^{2} .
$$

Comparing the predictions for the MSD in Eq. (19) and the MSSS in Eq. (24), we see that $\left\langle\tau_{a t}^{2}\right\rangle / \mu^{2} \sim\left\langle u_{a t}^{2}\right\rangle / a_{\text {lat }}^{2}$, which is expected considering the elastic nature of the 
problem at hand. In addition, we highlight that both quantities scale linearly with $\Delta \varepsilon^{2}$, showing the critical influence of this quantity to characterize random alloys, as already pointed out by previous authors [17, 24].

The real-space method described in this section enables to derive compact expressions for the MSD and MSSS where the influence of the atomic lattice is incorporated through an infinite sum that can be computed numerically. However, this real space method remains limited to simple cases. In particular, incorporating the influence of anisotropic elasticity would be challenging because the displacement and stress fields around an inclusion are no longer analytical [39, 49]. Also, we could in principle extend the real-space approach to investigate spatial correlations of the displacement and stress fields. However, the latter would be written in terms of intertwined sums, difficult to analyze. We will see in the following section and in Part II of this article that a Fourier-based approach may be better suited to anisotropic elasticity and spatial correlations because it yields simple analytical expressions easier to interpret.

\section{Fourier space method}

We introduce here a Fourier method based on the microelasticity theory [38, 39]. In order to overcome the limitations of the real space method and obtain simple analytical expressions for the MSD and MSSS as well as for spatial correlations, we introduce two modifications to the model: (1) we assume that the eigenstrains related to each inclusion are spread spatially following a Gaussian shape function of width $a$ and (2) we average the displacements and stresses over the entire continuum space and not only at the atomic sites. As a result, the MSD and MSSS no longer depend on the crystalline structure but only on the atomic volume, the elastic parameters and the new free parameter $a$. The latter may however be set by comparison with the real-space results.

\subsection{Inclusion field}

In contrast with the real space method described in section 2, we consider here that the eigenstrains are spatially smeared with a Gaussian spreading function defined as

$$
f(\boldsymbol{r})=\frac{v_{a t}}{(2 \pi)^{3 / 2} a^{3}} \exp \left(-\frac{|\boldsymbol{r}|^{2}}{2 a^{2}}\right),
$$

such that the inclusion volume identifies with the atomic volume $\left(v_{a t}=\int_{V} \boldsymbol{d} \boldsymbol{r} f(\boldsymbol{r})\right)$. We note $\theta_{\alpha}(\boldsymbol{r})$, the inclusion field associated with each element $\alpha$ :

$$
\theta_{\alpha}(\boldsymbol{r})=\sum_{n=1}^{N} P_{\alpha}(n) f\left(\boldsymbol{r}-\boldsymbol{R}_{\boldsymbol{n}}\right) .
$$


Microelasticity model of random alloys. Part I: mean square displacements and stresses 10

In the following, we denote Fourier transforms with the symbol $\sim$ and use the following convention:

$$
\begin{aligned}
\tilde{\theta}_{\alpha}(\boldsymbol{K}) & =\frac{1}{V} \int \boldsymbol{d} \boldsymbol{r} \theta_{\alpha}(\boldsymbol{r}) \exp (-i \boldsymbol{K} \cdot \boldsymbol{r}) \\
\theta_{\alpha}(\boldsymbol{r}) & =\sum_{\boldsymbol{K}} \tilde{\theta}_{\alpha}(\boldsymbol{K}) \exp (i \boldsymbol{K} \cdot \boldsymbol{r})
\end{aligned}
$$

where $\boldsymbol{K}=2 \pi\left(\frac{n_{x}}{L_{x}}, \frac{n_{y}}{L_{y}}, \frac{n_{z}}{L_{z}}\right)$ with $n_{x}, n_{y}, n_{z}$ three integers to satisfy the periodic boundary conditions applied to the volume $V$. The sum over the wave-vectors is infinite for a continuous medium. With the Gaussian spreading defined in Eq. (25), the Fourier transform of Eq. (26) is expressed as:

$$
\begin{aligned}
\tilde{\theta}_{\alpha}(\boldsymbol{K}) & =\tilde{f}(\boldsymbol{K}) \sum_{n} P_{\alpha}(n) \exp \left(-i \boldsymbol{K} . \boldsymbol{R}_{\boldsymbol{n}}\right) \\
& =\frac{v_{a t}}{V} e^{-\frac{a^{2} K^{2}}{2}} \sum_{n} P_{\alpha}(n) \exp \left(-i \boldsymbol{K} . \boldsymbol{R}_{\boldsymbol{n}}\right) .
\end{aligned}
$$

where the Fourier transform of the Gaussian smearing function $\tilde{f}(\boldsymbol{K})$ is also a Gaussian function in Fourier space.

\subsection{Displacement and stress fields}

In the microelasticity theory $[38,39]$, the displacement field is separated into homogeneous and inhomogeneous components. However, using the average alloy as reference ensures that the homogeneous displacement is zero. At equilibrium, the displacement field is then expressed as (repeated indices are summed):

$$
u_{i}(\boldsymbol{r})=\sum_{\boldsymbol{K} \neq \mathbf{0}}-i G_{i j}(\boldsymbol{K}) K_{l} C_{j l m n} \tilde{\epsilon}_{m n}^{0}(\boldsymbol{K}) e^{i \boldsymbol{K} \cdot \boldsymbol{r}},
$$

where $G_{i j}(\boldsymbol{K})$ is Green's function for elasticity in Fourier space and $\tilde{\varepsilon}_{m n}^{0}(\boldsymbol{K})=$ $\delta_{m n} \sum_{\alpha=1}^{N_{e l e m}} \varepsilon_{\alpha} \tilde{\theta}_{\alpha}(\boldsymbol{K})$, the Fourier transform of the eigenstrain field. The displacement field can be re-written as

$$
u_{i}(\boldsymbol{r})=\sum_{\alpha=1}^{N_{\text {elem }}} \varepsilon_{\alpha} \sum_{\boldsymbol{K} \neq 0} \Lambda_{i}(\boldsymbol{K}) \tilde{\theta}_{\alpha}(\boldsymbol{K}) e^{i \boldsymbol{K} \cdot \boldsymbol{r}},
$$

where $\Lambda_{i}(\boldsymbol{K})=-i G_{i j}(\boldsymbol{K}) K_{l} C_{j l m m}$ is an interaction kernel for displacements. Using the expression of $\tilde{\theta}_{\alpha}(\boldsymbol{K})$ in Eq. (28), this expression can be further rearranged as:

$$
u_{i}(\boldsymbol{r})=\sum_{n=1}^{N} \sum_{\alpha=1}^{N_{\text {elem }}} \varepsilon_{\alpha} P_{\alpha}(n) \mathcal{F}_{i}\left(\boldsymbol{r}-\boldsymbol{R}_{\boldsymbol{n}}\right)
$$


Microelasticity model of random alloys. Part I: mean square displacements and stresses 11 to recover a form analogous to Eq. (11) where the displacement at position $\boldsymbol{r}$ is written as a sum of contributions from all inclusion sites. We note that the function $\mathcal{F}_{i}(\boldsymbol{r})=\sum_{\boldsymbol{K} \neq 0} \tilde{f}(\boldsymbol{K}) \Lambda_{i}(\boldsymbol{K}) e^{i \boldsymbol{K} \cdot \boldsymbol{r}}$ differs from Eshelby solution defined in Eq. (10) for short distances because of the Gaussian spreading used here.

The microelasticity theory also yields an expression for the stress field:

$$
\sigma_{i j}(\boldsymbol{r})=\sum_{\alpha=1}^{N_{\text {elem }}} \varepsilon_{\alpha} \sum_{\boldsymbol{K} \neq \mathbf{0}}\left(\Xi_{i j}(\boldsymbol{K})-C_{i j k l} \delta_{k l}\right) \tilde{\theta}_{\alpha}(\boldsymbol{K}) e^{i \boldsymbol{K} \cdot \boldsymbol{r}}
$$

where $\Xi_{i j}(\boldsymbol{K})=C_{i j k l} G_{k p}(\boldsymbol{K}) K_{q} K_{l} C_{p q m m}$ is the interaction kernel for stresses. As for the displacements, Eq. (28) can be used to rewrite Eq. (32) and reveal the role of each atomic site $\boldsymbol{R}_{\boldsymbol{n}}$ on the stress at position $\boldsymbol{r}$ :

$$
\sigma_{i j}(\boldsymbol{r})=\sum_{n=1}^{N} \sum_{\alpha=1}^{N_{\text {elem }}} \varepsilon_{\alpha} P_{\alpha}(n)\left(\mathcal{G}_{i j}\left(\boldsymbol{r}-\boldsymbol{R}_{\boldsymbol{n}}\right)-C_{i j k l} \delta_{k l} f\left(\boldsymbol{r}-\boldsymbol{R}_{\boldsymbol{n}}\right)\right)
$$

where $\mathcal{G}_{i j}(\boldsymbol{r})=\sum_{\boldsymbol{K} \neq \mathbf{0}} \tilde{f}(\boldsymbol{K}) \Xi_{i j}(\boldsymbol{K}) e^{i \boldsymbol{K} \cdot \boldsymbol{r}}$. The second term represents the contribution of the eigenstrain to the stress. If we limit ourselves to isotropic elasticity and consider the shear stress components $(i \neq j), C_{i j k l} \delta_{k l}=0$ and we have $\S$

$$
\tau_{i j}(\boldsymbol{r})=\sum_{n=1}^{N} \sum_{\alpha=1}^{N_{\text {elem }}} \varepsilon_{\alpha} P_{\alpha}(n) \mathcal{G}_{i j}\left(\boldsymbol{r}-\boldsymbol{R}_{\boldsymbol{n}}\right)
$$

which is analogous to Eq.(20).

\subsection{Mean square displacement}

From Eq. (30), we have

$$
u_{i}(\boldsymbol{r}) \overline{u_{i}(\boldsymbol{r})}=\sum_{\boldsymbol{K} \neq \mathbf{0}} \sum_{\boldsymbol{K}^{\prime} \neq \mathbf{0}} \Lambda_{i}(\boldsymbol{K}) \overline{\Lambda_{i}\left(\boldsymbol{K}^{\prime}\right)} \sum_{\alpha=1}^{N_{\text {elem }}} \sum_{\beta=1}^{N_{\text {elem }}} \varepsilon_{\alpha} \varepsilon_{\beta} \tilde{\theta}_{\alpha}(\boldsymbol{K}) \overline{\tilde{\theta}_{\beta}\left(\boldsymbol{K}^{\prime}\right)} e^{i\left(\boldsymbol{K}-\boldsymbol{K}^{\prime}\right) \cdot \boldsymbol{r}} .
$$

We first average over the entire space and denote this average as $\langle\cdot\rangle_{s}$ :

$$
\left\langle u_{i}^{2}\right\rangle_{s}=\sum_{\boldsymbol{K} \neq \mathbf{0}} \sum_{\boldsymbol{K}^{\prime} \neq \mathbf{0}} \Lambda_{i}(\boldsymbol{K}) \overline{\Lambda_{i}\left(\boldsymbol{K}^{\prime}\right)} \sum_{\alpha=1}^{N_{\text {elem }}} \sum_{\beta=1}^{N_{\text {elem }}} \varepsilon_{\alpha} \varepsilon_{\beta} \tilde{\theta}_{\alpha}(\boldsymbol{K}) \overline{\tilde{\theta}_{\beta}\left(\boldsymbol{K}^{\prime}\right)} \frac{1}{V} \int e^{i\left(\boldsymbol{K}-\boldsymbol{K}^{\prime}\right) \cdot \boldsymbol{r}} \boldsymbol{d r}
$$

Using the fact that $\frac{1}{V} \int e^{i\left(\boldsymbol{K}-\boldsymbol{K}^{\prime}\right) \cdot \boldsymbol{r}} \boldsymbol{d} \boldsymbol{r}=\delta\left(\boldsymbol{K}-\boldsymbol{K}^{\prime}\right)$, we have:

$\S$ we use the symbol $\tau$ for the shear components of the stress 
Microelasticity model of random alloys. Part I: mean square displacements and stresses 12

$$
\left\langle u_{i}^{2}\right\rangle_{s}=\sum_{\boldsymbol{K} \neq \mathbf{0}}\left|\Lambda_{i}(\boldsymbol{K})\right|^{2} \sum_{\alpha=1}^{N_{\text {elem }}} \sum_{\beta=1}^{N_{\text {elem }}} \varepsilon_{\alpha} \varepsilon_{\beta} \tilde{\theta}_{\alpha}(\boldsymbol{K}) \overline{\tilde{\theta}_{\beta}(\boldsymbol{K})} .
$$

We wish to insist here on the fact that Eq. (37) is obtained after averaging the displacements over the entire space and not only on the atomic sites as in the real space approach described in section 2. This spatial average is necessary in order to keep the computation tractable analytically.

The next step consists in averaging over realizations of the random occupancy field (denoted by $\langle\cdot\rangle_{\mathcal{R}}$ ):

$$
\left\langle\left\langle u_{i}^{2}\right\rangle_{s}\right\rangle_{\mathcal{R}}=\sum_{\boldsymbol{K} \neq \mathbf{0}}\left|\Lambda_{i}(\boldsymbol{K})\right|^{2} \sum_{\alpha=1}^{N_{\text {elem }}} \sum_{\beta=1}^{N_{\text {elem }}} \varepsilon_{\alpha} \varepsilon_{\beta}\left\langle\tilde{\theta}_{\alpha}(\boldsymbol{K}) \overline{\tilde{\theta}_{\beta}(\boldsymbol{K})}\right\rangle_{\mathcal{R}}
$$

As shown in Appendix A, the term $\sum_{\alpha} \sum_{\beta} \varepsilon_{\alpha} \varepsilon_{\beta}\left\langle\tilde{\theta}_{\alpha}(\boldsymbol{K}) \bar{\theta}_{\beta}(\boldsymbol{K})\right\rangle_{\mathcal{R}}$ can be simplified to:

$$
\sum_{\alpha=1}^{N_{\text {elem }}} \sum_{\beta=1}^{N_{\text {elem }}} \varepsilon_{\alpha} \varepsilon_{\beta}\left\langle\tilde{\theta}_{\alpha}(\boldsymbol{K}) \overline{\tilde{\theta}_{\beta}(\boldsymbol{K})}\right\rangle_{\mathcal{R}}=\frac{v_{a t} \Delta \varepsilon^{2}}{V} e^{-a^{2} K^{2}}
$$

Combining all three spatial directions, we obtain the variance of the displacement field, that represents the MSD:

$$
\left\langle u^{2}\right\rangle=\frac{v_{a t} \Delta \varepsilon^{2}}{V} \sum_{\boldsymbol{K} \neq \mathbf{0}} \sum_{d=1}^{3}\left|\Lambda_{d}(\boldsymbol{K})\right|^{2} e^{-a^{2} \boldsymbol{K}^{2}},
$$

where, for simplicity reasons, brackets without subscripts are used for space and ensemble averages $\left(\langle\cdot\rangle=\left\langle\langle\cdot\rangle_{s}\right\rangle_{\mathcal{R}}\right)$. Finally, considering the limit of an infinite system, we can transform the sum over $\boldsymbol{K}$ vectors into an integral and obtain:

$$
\left\langle u^{2}\right\rangle=\frac{v_{a t} \Delta \varepsilon^{2}}{(2 \pi)^{3}} \int \sum_{d=1}^{3}\left|\Lambda_{d}(\boldsymbol{K})\right|^{2} e^{-a^{2} \boldsymbol{K}^{2}} \boldsymbol{d} \boldsymbol{K} .
$$

Eq. (41) shows that the mean square displacement is directly proportional to $\Delta \varepsilon^{2}$, for any anisotropic elastic medium. In the special case of an elastically isotropic medium, the kernel $\Lambda_{d}(\boldsymbol{K})$ takes a simple expression [39]:

$$
\Lambda_{d}(\boldsymbol{K})=-i \frac{1+\nu}{1-\nu} \frac{K_{d}}{\boldsymbol{K}^{2}}
$$

and the integral in Eq. (41) can be computed analytically to yield: 


$$
\left\langle u^{2}\right\rangle=\frac{v_{a t} \Delta \varepsilon^{2}}{4 \pi^{3 / 2} a}\left(\frac{1+\nu}{1-\nu}\right)^{2} .
$$

Eq. (43) shows that the MSD is inversely proportional to the spreading distance $a$ : more peaked eigenstrain distributions lead to larger displacements in their vicinity. By contrast, in the limit $a \rightarrow+\infty$, the eigenstrains spread out over the entire space and the fluctuations converge to zero.

We note here that Eq. (43) does not depend on the crystalline structure but only the atomic volume, because it was obtained through an average over the continuum space. In contrast, the real-space approach detailed in section 2 depends on the atomic structure. However, comparison between Eqs. (43) and (19) shows that crystallography enters only through a geometric prefactor.

To bridge the Fourier- and real-space approaches, we can adjust the parameter $a$ to incorporate the influence of the crystalline lattice. Equating Eq. (43) with Eq. (19), we find for a FCC lattice:

$$
a_{u}=\frac{4 \sqrt{\pi}}{25.3} a_{\text {lat }} \simeq 0.28 a_{\text {lat }}
$$

where the subscript $u$ denotes that this quantity was adjusted on the value of the MSD. Eq. (44) shows that $a_{u}$ is smaller than the lattice spacing $a_{l a t}$, which ensures that the Gaussian distributions on different atoms do not overlap.

\subsection{Mean square shear stress}

We now focus on the stresses. From Eq. (33), we have:

$$
\begin{aligned}
\sigma_{i j}(\boldsymbol{r}) & =\sum_{n=1}^{N}\left\{\sum_{\alpha=1}^{N_{\text {elem }}} \varepsilon_{\alpha} P_{\alpha}(n) \mathcal{G}_{i j}\left(\boldsymbol{r}-\boldsymbol{R}_{\boldsymbol{n}}\right)\right\}-C_{i j k l} \delta_{k l} \sum_{n=1}^{N}\left\{\sum_{\alpha=1}^{N_{\text {elem }}} \varepsilon_{\alpha} P_{\alpha}(n) f\left(\boldsymbol{r}-\boldsymbol{R}_{\boldsymbol{n}}\right)\right\} \\
& =\sum_{n=1}^{N} \eta_{n}-C_{i j k l} \delta_{k l} \sum_{n=1}^{N} \eta_{n}^{\prime} .
\end{aligned}
$$

The first sum in the above equation follows a normal distribution in the limit of large $N$ because the random variables $\eta_{n}$ satisfy the conditions of Lyapunov's central-limit theorem [47]. On the other hand, if $a$ is on the order to the lattice parameter or below, the Gaussian smearing $f(\boldsymbol{r})$ decreases too rapidly for the random variable $\eta_{n}^{\prime}$ to converge to a Gaussian approximation. Instead, $\eta_{n}^{\prime}$ follows a multimodal distribution with peaks corresponding to each value of $\varepsilon_{\alpha}$. Therefore internal stresses do not follow in general a normal distribution. An exception are shear stresses in an isotropic elastic medium, where $C_{i j k l} \delta_{k l}=0$ and the shear stresses follow the normal distribution given by the first sum in Eq. (45). In this case, the elastic kernel $\Xi_{i j}(\boldsymbol{K})$ reduces to: 
Microelasticity model of random alloys. Part I: mean square displacements and stresses14

$$
\Xi_{i j}(\boldsymbol{K})=2 \mu \frac{1+\nu}{1-\nu} \frac{K_{i} K_{j}}{K^{2}} .
$$

Following the same steps as in Section 3.3, the MSSS can be computed in the limit of an infinite system:

$$
\left\langle\tau^{2}\right\rangle=\frac{v_{a t} \Delta \varepsilon^{2}}{(2 \pi)^{3}} 4 \mu^{2}\left(\frac{1+\nu}{1-\nu}\right)^{2} \int \frac{K_{i}^{2} K_{j}^{2}}{K^{4}} e^{-a^{2} \boldsymbol{K}^{2}} \boldsymbol{d} \boldsymbol{K},
$$

which is independent of the $i$ and $j$ indices because of the isotropy of the medium and is expressed as

$$
\left\langle\tau^{2}\right\rangle=\frac{v_{a t} \Delta \varepsilon^{2} \mu^{2}}{30 \pi^{3 / 2} a^{3}}\left(\frac{1+\nu}{1-\nu}\right)^{2} .
$$

The MSSS is proportional to $\mu^{2}$ as expected, and to $1 / a^{3}$. Mean square stresses are therefore more sensitive to the Gaussian spreading than the mean square displacement (see Eq. (43)). We note that, as for the MSD, Eq. (48) is averaged over the continuum space and does not depend on the atomic structure.

As for the MSD, we can equate Eq. (48) with Eq. (24) to set the parameter $a$ and incorporate the effect of the underlying atomic lattice:

$$
a_{\tau}=\left(\frac{16 \sqrt{\pi}}{2434.8}\right)^{1 / 3} a_{\text {lat }}=0.23 a_{\text {lat }} .
$$

This value differs from $a_{u}=0.28 a_{\text {lat }}$, showing that the crystalline lattice influences differently the MSD and MSSS. This is explained by the fact that the displacement and stress fields decrease respectively as $1 / r^{2}$ and $1 / r^{3}$ around inclusions, changing the sum over lattice sites involved in Eq. (19) and Eq. (24).

\section{Comparison with molecular statics simulations}

Atomistic calculations provide an ideal tool to validate the theory and the analytical expressions derived in this work. To test the theory, we first consider a binary LennardJones model system with a small size effect and no chemical interactions between species. Next, we use an EAM potential for Al-Mg [50] that incorporates more complex atomic interactions.

As discussed in Part II [51], the system size influences significantly the results obtained for the mean-square displacement because of the long-range correlations between displacements. To reduce these finite size effects to a minimum, we performed simulations in large simulation cells containing 16384000 atoms (cubic cell of size $\left.\left(160 a_{\text {lat }}\right)^{3}\right)$. The atomic displacements are measured with respect with the average lattice and the atomic stress are obtained from the virial method. Both quantities are averaged 
over the entire system to yield atomistic estimates of the mean-square displacement and shear stress.

A meaningful comparison between these quantities and the solutions of the elastic model requires to estimate the elastic parameters of Eq. (19) and Eq. (24). These parameters (namely the elastic constants $\mu, \nu$, the eigenstrains $\varepsilon_{\alpha}$, the lattice parameter $a_{\text {lat }}$ and the average atomic volume $v_{a t}$ ) are computed beforehand using small simulation cells containing 4,000 atoms.

All atomistic calculations are performed with Lammps software [52].

\subsection{Lennard-Jones binary system}

We first consider a FCC binary alloy $\mathrm{A}_{c_{A}} \mathrm{~B}_{c_{B}}$ (with $c_{A}+c_{B}=1$ ) modeled with a LennardJones (LJ) interatomic potential. To introduce a small size mismatch, we use a shifted potential:

$$
E_{L J}(\Delta, r)=4 \varepsilon_{L J}\left[\left(\frac{\sigma_{L J}}{r-\Delta}\right)^{12}-\left(\frac{\sigma_{L J}}{r-\Delta}\right)^{6}\right]
$$

and define the atomic interactions between pairs of atoms as:

$$
\begin{aligned}
& E_{A A}(r)=E_{A A}(0, r), \\
& E_{A B}(r)=E_{A B}(\Delta / 2, r), \\
& E_{B B}(r)=E_{B B}(\Delta, r) .
\end{aligned}
$$

With $\Delta \ll \sigma_{L J}$, this definition allows to introduce a size mismatch without modifying significantly the elastic properties of the alloy. LJ parameters, $\varepsilon_{L J}=0.50722 \mathrm{eV}$ and $\sigma_{L J}=2.57366 \AA$, were employed to reproduce physical properties similar to a transition metal. In addition, a small value of $\Delta=0.01 \AA \simeq 3.9 \cdot 10^{-3} \sigma_{L J}$ was used. We checked numerically that the random AB alloys modeled with this potential satisfy Vegard's law with an average equilibrium lattice spacing linearly dependent on the composition:

$$
a_{\text {lat }}=a_{A} c_{A}+a_{B} c_{B}
$$

with $a_{A}=3.9744 \AA$ and $a_{B}=3.9896 \AA$, the equilibrium lattice parameters of the pure A and $B$ lattices respectively. We also computed the eigenstrains $\varepsilon_{A}$ and $\varepsilon_{B}$ from atomistic calculations using the definition given in Eq. (7) and following the method described in Ref. [21]. The result is shown as circles in Fig. 2.a. The linear dependence of the eigenstrains on composition visible in this figure is expected since, if we assume Vegard's law and account for the fact that $\left|a_{B}-a_{A}\right| \ll a_{A}$, Eq. (7) simplifies to:

$$
\begin{aligned}
\varepsilon_{A} & =-c_{B} \frac{a_{B}-a_{A}}{a_{A}} \\
\varepsilon_{B} & =c_{A} \frac{a_{B}-a_{A}}{a_{A}}
\end{aligned}
$$


Microelasticity model of random alloys. Part I: mean square displacements and stresses16

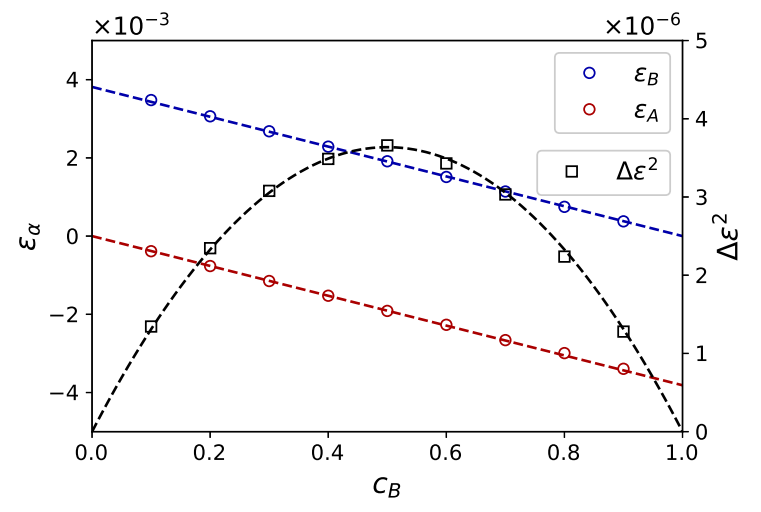

(a)

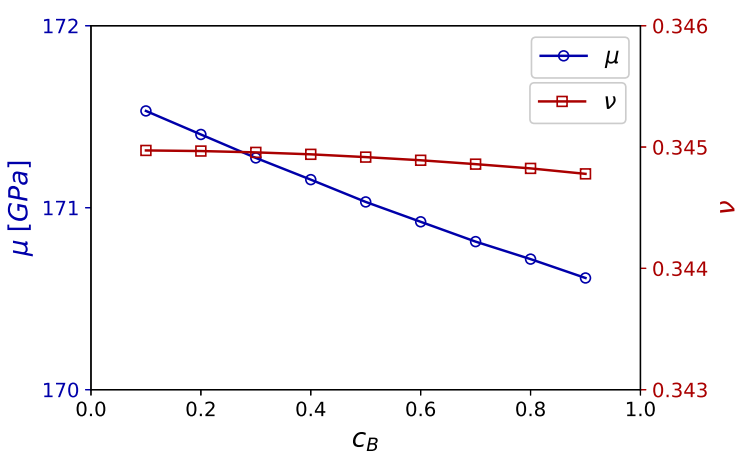

(b)

Figure 2. Lennard-Jones model: (a) Eigenstrains $\varepsilon_{A}$ and $\varepsilon_{B}$ and variance $\Delta \varepsilon^{2}$ (see Eq. (17)) as a function of composition. Atomistic data (open symbols) are compared to the predictions of the model (dashed lines). (b) Isotropic shear modulus and Poisson's ratio obtained using Bacon-Scattergood average.

These linear functions are shown as dashed lines in Fig. 2.a and perfectly match the numerical data. As a result, the variance of the eigenstrain, also shown in Fig. 2.a, is a parabolic function given by:

$$
\Delta \varepsilon^{2}=c_{A} \varepsilon_{A}^{2}+c_{B} \varepsilon_{B}^{2}=\left(\frac{a_{B}-a_{A}}{a_{A}}\right)^{2} c_{B}\left(1-c_{B}\right) .
$$

We computed the average elastic constants of the $\mathrm{AB}$ random alloys as a function of their composition. Isotropic elastic constants $\mu$ and $\nu$ were obtained using the BaconScattergood average $[53,54]$ and are shown in Fig. 2.b. As expected with the potential used here, the shear modulus and Poisson's ratio do not vary significantly (less than $1 \%$ ) over the composition range. In the following, we will consider $\mu$ and $\nu$ independent of the composition with values corresponding to the $\mathrm{A}_{0.5} \mathrm{~B}_{0.5}$ alloy: $\mu=171.1 \mathrm{GPa}$ and $\nu=0.345$.

Fig. 3.a displays with round symbols the MSD computed on the atomistic model as a function of the alloy composition. The MSD is obtained from the difference between the positions of the atoms and their lattice sites in the average alloy. We also report in Fig. 3.a as a dashed curve, the MSD predicted by Eq. (19) or equivalently, by Eq. (43) using $a=0.28 a_{\text {lat }}$. The elastic model reproduces very accurately the composition dependence of the mean-square displacement, without fitting parameters. We note that in this model alloy, the composition dependence of the mean square displacements depends only on the variance $\Delta \varepsilon^{2}$ and thus shows the same parabolic behavior.

Fig. 3.b displays the MSSS as a function of composition. The atomistic results compare well with the analytical prediction of Eq. (24) (or equivalently, Eq. (48) with $a=0.23 a_{\text {lat }}$ ) shown as a dashed line. The $15 \%$ difference between both results can be explained by the elastic anisotropy of the LJ system and the inadequacy of the virial method to compute accurately atomic stresses as demonstrated in the literature 
Microelasticity model of random alloys. Part I: mean square displacements and stresses 17

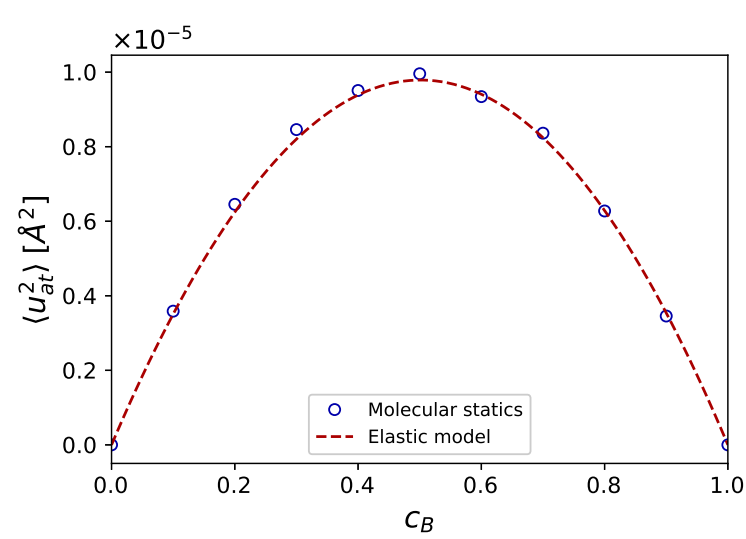

(a)

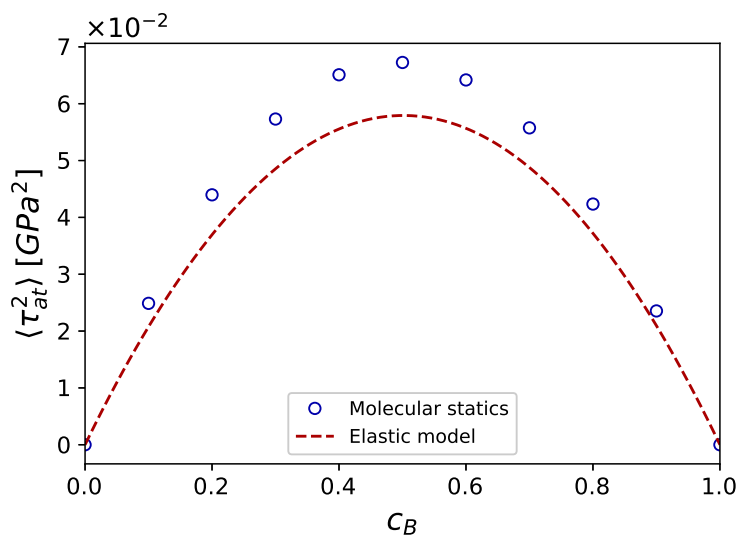

(b)

Figure 3. Lennard-Jones model: mean square displacements (a) and shear stresses (b) as a function of composition. Atomistic data (open symbols) are compared to the predictions of the real space approach (Eqs. (19) and (24)) (full lines).

$[55,56,57]$.

\subsection{Al-Mg system}

To go beyond the LJ model, we considered more realistic Al-Mg random alloys modeled with the embedded atom method (EAM) potential of Liu et al. [50]. In this system, the size difference between $\mathrm{Al}$ and $\mathrm{Mg}$ atoms remains reasonable and the elastic constants display a moderate anisotropy, making it a good candidate to test the elastic model.

As before, we start by computing the elastic properties of the alloys as a function of their composition. Fig. 4.a displays the eigenstrains $\varepsilon_{A l}$ and $\varepsilon_{M g}$. In contrast with the LJ model, the eigenstrains are no longer linear functions of the composition, revealing a deviation from Vegard's law. Due to this non-linearity, the variance $\Delta \varepsilon^{2}$ shown with square symbols is significantly different than the parabolic shape of Fig. 2.a. Fig. 4.b shows the shear modulus and Poisson's ratio as a function of alloy composition. While Poisson's ratio varies only between 0.32 and 0.36 , the shear modulus depends significantly on composition and drops by $40 \%$ between $\mathrm{Al}_{0.9} \mathrm{Mg}_{0.1}$ and $\mathrm{Al}_{0.6} \mathrm{Mg}_{0.4}$.

The MSD and MSSS measured from molecular statics are shown with round symbols in Fig. 5.a and b. They are compared to the prediction of Eq. (19) (or Eq. (43)) and Eq. (24) (or Eq. (48)) where the eigenstrains and elastic constants are taken from Fig. 4.

Despite the complexity of the interatomic potential and the significant size difference between $\mathrm{Al}$ and $\mathrm{Mg}$ atoms, the prediction of our elastic approach reproduces very well the MSD and very satisfactorily the MSSS without adjusting parameters. 
Microelasticity model of random alloys. Part I: mean square displacements and stresses 18

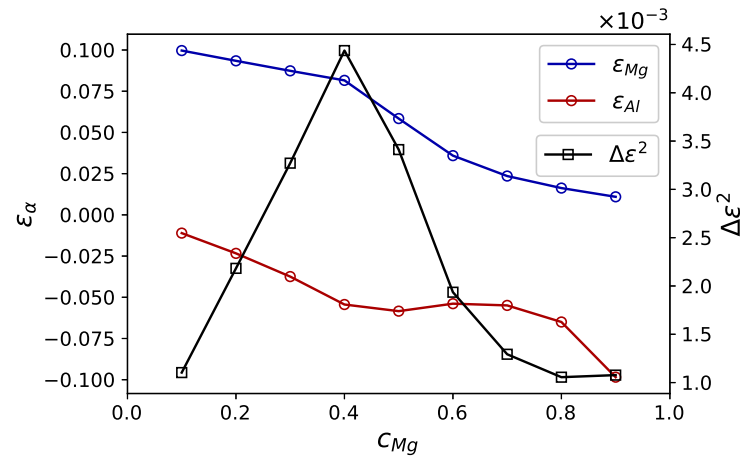

(a)

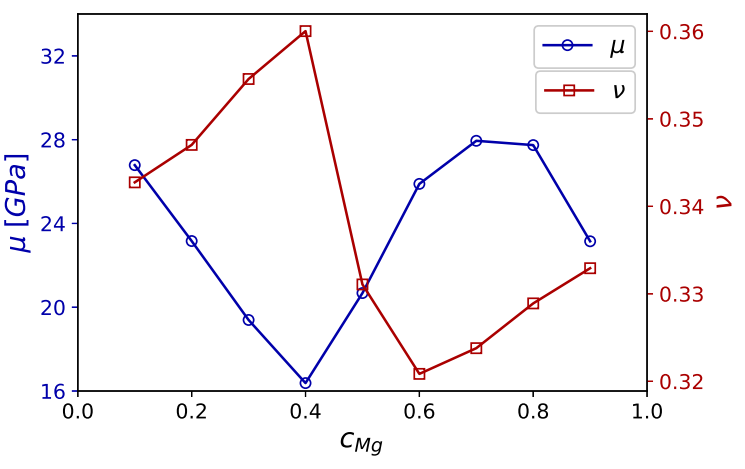

(b)

Figure 4. Al-Mg alloy: (a) eigenstrains $\varepsilon_{A l}$ and $\varepsilon_{M g}$ and variance $\Delta \varepsilon^{2}$, (b) isotropic shear modulus and Poisson's ratio as a function of the composition. Atomistic data are shown as open symbols.

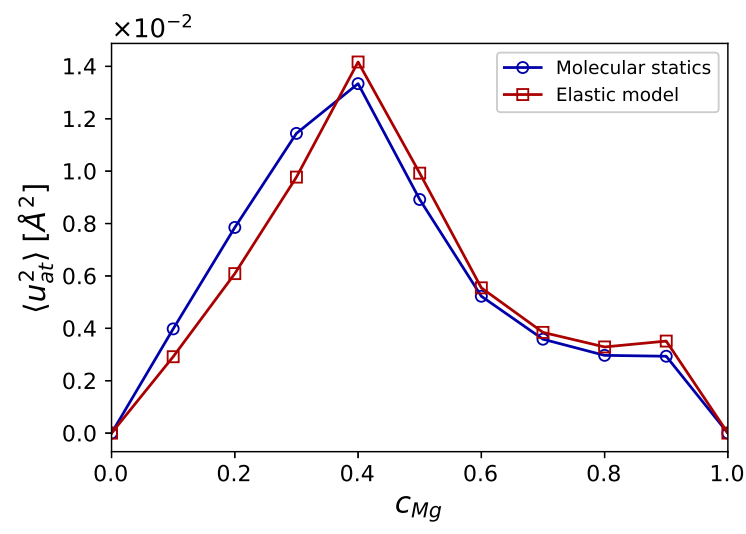

(a)

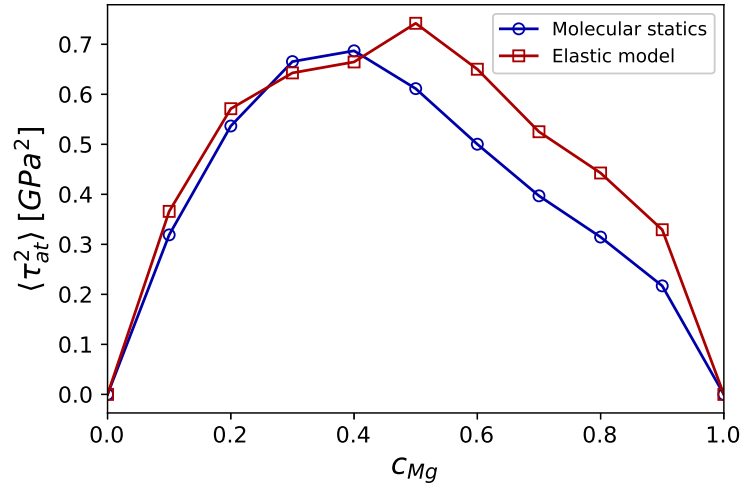

(b)

Figure 5. Al-Mg alloy: mean square displacements (a) and shear stresses (b) as a function of composition. Atomistic data (open circles) are compared to the predictions of the model (open squares).

\section{Discussion}

We proposed two different elastic approaches to obtain the mean square displacement and the mean square shear stress by treating an alloy as an elastic medium and the atoms as elastic inclusions. In the real-space approach detailed in section 2 , the atomic displacements and stresses are computed as sums of the contributions of all surrounding atoms and the MSD and MSSS are expressed as infinite sums over the lattice sites. In section 3, we also propose a Fourier approach based on the microelasticity theory, where the MSD and MSSS are obtained after averaging over the entire space and not only at the atomic positions. As a consequence, these quantities do not depend on the crystalline structure, but only on the atomic density. Therefore, the results of the Fourier approach can be seen as long wavelengths approximations where the role of the 
crystalline lattice entering only the short wave-lengths terms has been discarded. Apart from this, both real and Fourier approaches yield similar expressions for the MSD (see Eq. (19) and Eq. (43)) and MSSS (see Eq. (24) and Eq. (48)). In particular, they are all proportional to the variance of the eigenstrains, $\Delta \varepsilon^{2}$, which reduces to the square of the size misfit parameter, $\delta^{2}$, if the alloy satisfies Vegard's law. We therefore recover a linear relation similar to the scaling proposed in Eq. (3) and observed by means of ab initio calculations in Ref. [13] but different from that investigated in Ref. [18].

Interestingly, combining the real or equivalently spectral expressions of the MSSS with the scaling relation in Eq. (4) derived in the framework of the depinning transition [34] and assuming Vegard's law, we obtain the following relation for the critical resolved shear stress at $0 \mathrm{~K}$ :

$$
\tau_{y 0} \sim \frac{\left\langle\tau^{2}\right\rangle^{2 / 3}}{\Gamma^{1 / 3}} \sim \frac{1}{\Gamma^{1 / 3}}\left(\mu \frac{1+\nu}{1-\nu}\right)^{4 / 3} \delta^{4 / 3},
$$

revealing exactly the same scaling as in Varvenne's model (see Eq. (2)) between the elastic constants $\mu$ and $\nu$, the dislocation line tension $\Gamma$ and the size mismatch parameter. This demonstrates the equivalence between the energetic considerations used in Varvenne's model and the stress considerations at the basis of the depinning transition theory. This agreement is a consequence of Betti's reciprocal theorem [58], which states the equivalence between both viewpoints: (i) looking at the interactions between the stress field of a dislocation and solutes of different sizes as considered in Ref. $[8,9,17]$ and (ii) looking at the dislocation interaction with the stress field emerging from the solid solution as considered here.

We also note that since in the elastic models $\left\langle\tau^{2}\right\rangle / \mu^{2} \sim\left\langle u^{2}\right\rangle / a_{\text {lat }}^{2}$, we have necessarily $\tau_{y 0} \sim\left\langle\tau^{2}\right\rangle^{2 / 3} \sim\left\langle u^{2}\right\rangle^{2 / 3}$. This scaling is different from the linear relation between yield stress and root mean square displacement proposed by Okamoto et al. [31]. However, the yield stresses in Ref. [31] are experimental data extrapolated to $0 \mathrm{~K}$, which may involve error bars while a $4 / 3$ scaling is not significantly different from a linear scaling, especially for the limited range of the data set considered in Ref. [31].

Both the real- and Fourier-space approaches presented here have their pros and cons. The real-space model has the advantage of being parameter free but it does not allow for a straightforward nor insightful extension to anisotropic elasticity since the displacement and stress fields around an inclusion are then not analytical [39, 49]. Extension to spatial correlations is also not relevant because it leads to complicated intertwined sums. By way of contrast, the spectral model can be readily extended to anisotropic crystals and can be used to obtain compact analytical expressions for the spatial correlations of the displacement and stress fields. This justifies its use in Part II of this article. The main drawback of the spectral method is to rely on the adjustment of an internal parameter, $a$. This parameter can be adjusted on the real-space geometric prefactor to account in an effective manner for the effect of the crystalline lattice. Interestingly, this leads to slightly different expressions of $a$ depending on whether we consider the MSD (Eq. 44) or MSSS (Eq. 49). The reason is that the 
discretization of the real-space crystalline lattice as well as the Gaussian smearing used in the spectral approach affect differently the displacements and stresses because of their different spatial dependencies.

The comparison between the elastic model and atomistic calculations in the case of Al-Mg alloys reveals the importance of using composition-dependent parameters as input of the elastic model. In particular, the non-linearity of the eigenstrains and elastic constants evidenced in Fig. 4 is essential to reproduce the MSD and MSSS of Fig. 5. In other words, these results suggest that, in general, concentrated alloys can not be considered as linear interpolations of pure metals and assuming Vegard's law may lead to significant errors in predicting quantities such as the MSD and MSSS and in turn, the yield stress of the alloy.

Some differences were observed between the model predictions and atomistic calculations that arise from the simplicity of the model. An important limitation consists in modeling atoms as purely dilatational inclusions while the eigenstrains may include shear components. The eigenstrain tensor may also vary with the local environment, which could be readily included by incorporating the role of different environments as done in Ref. [17]. Another simplifying assumption consists in neglecting the effect of chemical interactions between atoms that can contribute to the atomic displacements and stresses. Depending on the system, these chemical effects can impede the dislocation and contribute significantly to solute strengthening as shown recently by Nag and Curtin [59]. Another related issue is the role of the elastic constant mismatch between species, which cannot be incorporated easily in this type of elastic model even though it is considered to play an significant role in solid solution strengthening $[7,8,10]$.

\section{Conclusion}

In this article, we developed two elastic models based respectively on real- and Fourier-space approaches. Interestingly, both approaches show that the mean square displacement and the mean square shear stress are proportional to the variance of the eigenstrains $\Delta \varepsilon^{2}=\sum_{\alpha} c_{\alpha} \varepsilon_{\alpha}^{2}$, which appears as the main parameter to describe displacements and stresses in random solid solutions. In Section 4, we compared the prediction of the models to atomistic calculations and found that, despite their simplifying assumptions (isotropic elasticity, no chemical contribution, pure dilatational eigenstrains), the elastic models reproduce without fitting parameters the compositiondependent MSD and MSSS obtained from molecular statics. We have however seen that it is necessary to go beyond Vegard's law to reproduce the MSD and MSSS, even in a simple binary alloy as AlMg. Moreover, preliminary results suggest that incorporating eigenstrain tensors that are not purely dilatational and depend on the local environment may be required to predict the MSD and MSSS accurately in more complex alloys such as high entropy alloys. This constitutes the main perspective of this work. 
Microelasticity model of random alloys. Part I: mean square displacements and stresses21

\section{Acknowledgment}

P.A.G wishes to acknowledge the financial support of IDEXLYON Impulsion project from the University of Lyon in the framework of the Programme Investissements d'Avenir (ANR-16-IDEX-005) grant from the University of Lyon IDEX. P.A.G also wishes to thank his wife for the quality time spent during the Covid-19 lockdown.

\section{Appendix A. Computation of $\sum_{\alpha} \sum_{\beta} \varepsilon_{\alpha} \varepsilon_{\beta}\left\langle\tilde{\theta}_{\alpha}(\boldsymbol{K}) \overline{\tilde{\theta}_{\beta}(\boldsymbol{K})}\right\rangle_{\mathcal{R}}$}

From the definition of $\tilde{\theta}_{\alpha}(\boldsymbol{K})$ in Eq. (28), we have

$$
\tilde{\theta}_{\alpha}(\boldsymbol{K}) \overline{\tilde{\theta}_{\beta}(\boldsymbol{K})}=\left(\frac{v_{a t}}{V}\right)^{2} e^{-a^{2} K^{2}} \sum_{n=1}^{N} \sum_{m=1}^{N} P_{\alpha}(n) P_{\beta}(m) \exp \left(i \boldsymbol{K} .\left(\boldsymbol{R}_{\boldsymbol{n}}-\boldsymbol{R}_{\boldsymbol{m}}\right)\right),
$$

where the sums run over all the inclusion sites. We consider first the case $\alpha=$ $\beta$. Averaging over realizations and using the fact that the properties of $P_{\alpha}(n)$ are independent of $n$, we find:

$$
\begin{aligned}
\left\langle\left|\tilde{\theta}_{\alpha}(\boldsymbol{K})\right|^{2}\right\rangle_{\mathcal{R}} & =\left(\frac{v_{a t}}{V}\right)^{2} e^{-a^{2} K^{2}}\left[N\left\langle P_{\alpha}^{2}\right\rangle+\left\langle P_{\alpha}\right\rangle^{2} \sum_{n \neq m} \exp \left(i \boldsymbol{K} .\left(\boldsymbol{R}_{\boldsymbol{n}}-\boldsymbol{R}_{\boldsymbol{m}}\right)\right)\right] \\
& =\left(\frac{v_{a t}}{V}\right)^{2} e^{-a^{2} K^{2}}\left[N\left(\left\langle P_{\alpha}^{2}\right\rangle-\left\langle P_{\alpha}\right\rangle^{2}\right)+\left\langle P_{\alpha}\right\rangle^{2} \sum_{n, m} \exp \left(i \boldsymbol{K} .\left(\boldsymbol{R}_{\boldsymbol{n}}-\boldsymbol{R}_{\boldsymbol{m}}\right)\right)\right] \\
& =\left(\frac{v_{a t}}{V}\right)^{2} e^{-a^{2} K^{2}}\left[N c_{\alpha}\left(1-c_{\alpha}\right)+c_{\alpha}^{2}|S(\boldsymbol{K})|^{2}\right]
\end{aligned}
$$

where $S(\boldsymbol{K})=\sum_{n} \exp \left(i \boldsymbol{K} . \boldsymbol{R}_{\boldsymbol{n}}\right)$ is the structure factor of the inclusion lattice. In the case $\alpha \neq \beta$, using Eq. (6), we have:

$$
\begin{aligned}
\left\langle\tilde{\theta}_{\alpha}(\boldsymbol{K}) \tilde{\tilde{\theta}}_{\beta}(\boldsymbol{K})\right\rangle_{\mathcal{R}} & =\left(\frac{v_{a t}}{V}\right)^{2} e^{-a^{2} K^{2}}\left\langle P_{\alpha}\right\rangle\left\langle P_{\beta}\right\rangle \sum_{n \neq m} \exp \left(i \boldsymbol{K} .\left(\boldsymbol{R}_{\boldsymbol{n}}-\boldsymbol{R}_{\boldsymbol{m}}\right)\right) \\
& =\left(\frac{v_{a t}}{V}\right)^{2} e^{-a^{2} K^{2}}\left[-N\left\langle P_{\alpha}\right\rangle\left\langle P_{\beta}\right\rangle+\left\langle P_{\alpha}\right\rangle\left\langle P_{\beta}\right\rangle|S(\boldsymbol{K})|^{2}\right] \\
& =\left(\frac{v_{a t}}{V}\right)^{2} e^{-a^{2} K^{2}}\left[-N c_{\alpha} c_{\beta}+c_{\alpha} c_{\beta}|S(\boldsymbol{K})|^{2}\right] .
\end{aligned}
$$

Combining Eq. (A.2) and (A.3), we find:

$$
\begin{array}{r}
\sum_{\alpha=1}^{N_{\text {elem }}} \sum_{\beta=1}^{N_{\text {elem }}} \varepsilon_{\alpha} \varepsilon_{\beta}\left\langle\tilde{\theta}_{\alpha}(\boldsymbol{K}) \overline{\tilde{\theta}_{\beta}(\boldsymbol{K})}\right\rangle_{\mathcal{R}}=\left(\frac{v_{a t}}{V}\right)^{2} e^{-a^{2} K^{2}}\left[N \sum_{\alpha=1}^{N_{\text {elem }}} \sum_{\beta=1}^{N_{\text {elem }}} \varepsilon_{\alpha} \varepsilon_{\beta}\left(c_{\alpha} \delta_{\alpha \beta}-c_{\alpha} c_{\beta}\right)\right. \\
\left.+|S(\boldsymbol{K})|^{2} \sum_{\alpha=1}^{N_{\text {elem }}} \sum_{\beta=1}^{N_{\text {elem }}} \varepsilon_{\alpha} \varepsilon_{\beta} c_{\alpha} c_{\beta}\right] .
\end{array}
$$


The first term between the square brackets yields the variance of the eigenstrains $\Delta \varepsilon^{2}$ since:

$$
\sum_{\alpha=1}^{N_{\text {elem }}} \sum_{\beta=1}^{N_{\text {elem }}} \varepsilon_{\alpha} \varepsilon_{\beta} c_{\alpha}\left(\delta_{\alpha \beta}-c_{\beta}\right)=\sum_{\alpha}^{N_{\text {elem }}} c_{\alpha} \varepsilon_{\alpha}^{2}-\left(\sum_{\alpha}^{N_{\text {elem }}} c_{\alpha} \varepsilon_{\alpha}\right)^{2}=\Delta \varepsilon^{2}
$$

Note that the average alloy assumption implies that $\sum_{\alpha} c_{\alpha} \varepsilon_{\alpha}=0$ and thus the variance simplifies to $\Delta \varepsilon^{2}=\sum_{\alpha} c_{\alpha} \varepsilon_{\alpha}^{2}$. In addition, this assumption also implies that the second term between the square brackets of Eq. (A.4), proportional to $\left(\sum_{\alpha} c_{\alpha} \varepsilon_{\alpha}\right)^{2}$, is zero. Finally, we have:

$$
\sum_{\alpha=1}^{N_{\text {elem }}} \sum_{\beta=1}^{N_{\text {elem }}} \varepsilon_{\alpha} \varepsilon_{\beta}\left\langle\tilde{\theta}_{\alpha}(\boldsymbol{K}) \overline{\tilde{\theta}_{\beta}(\boldsymbol{K})}\right\rangle_{\mathcal{R}}=\frac{v_{a t} \Delta \varepsilon^{2}}{V} e^{-a^{2} \boldsymbol{K}^{2}}
$$

\section{References}

[1] Y. Zhang, T.T. Zuo, Z. Tang, M.C. Gao, K.A. Dahmen, P.K. Liaw, and Z.P. Lu. Microstructures and properties of high-entropy alloys. Prog. Mater. Sci., 61:1-93, 2014.

[2] O.N. Senkov, D.B. Miracle, K.J. Chaput, and J.-P. Couzinié. Development and exploration of refractory high entropy alloys - A review. J. Mater. Res., 33(19):30923128, 2018.

[3] J.-W. Yeh. Recent progress in high entropy alloys. Ann. Chim. Sci. Mat, 31(6):633-648, 2006.

[4] O.N. Senkov, G.B. Wilks, D.B. Miracle, C.P. Chuang, and P.K. Liaw. Refractory highentropy alloys. Intermetallics, 18(9):1758-1765, 2010.

[5] Y. Zhang, X. Yang, and P.K. Liaw. Alloy design and properties optimization of highentropy alloys. JOM, 64(7):830-838, 2012.

[6] S.S. Sohn, A. Kwiatkowski da Silva, Y. Ikeda, F. Körmann, W. Lu, W.S. Choi, B. Gault, D. Ponge, J. Neugebauer, and D. Raabe. Ultrastrong medium-entropy single-phase alloys designed via severe lattice distortion. Adv. Mater., 31(8):1807142, 2019.

[7] R.L. Fleischer. Substitutional solution hardening. Acta Metall., 11(3):203-209, 1963.

[8] R. Labusch. A statistical theory of solid solution hardening. Phys. Stat. Sol. (b), 41(2):659-669, 1970.

[9] G.P.M. Leyson, W.A. Curtin, L.G. Hector, and C.F. Woodward. Quantitative prediction of solute strengthening in aluminium alloys. Nat. Mater., 9(9):750-755, 2010.

[10] I. Toda-Caraballo and P.E.J. Rivera-Díaz-del Castillo. Modelling solid solution hardening in high entropy alloys. Acta Mater., 85:14-23, 2015.

[11] Y. Zhang, Y.J. Zhou, J.P. Lin, G.L. Chen, and P.K. Liaw. Solid-solution phase formation rules for multi-component alloys. Adv. Eng. Mater., 10(6):534-538, 2008.

[12] Q. He and Y. Yang. On lattice distortion in high entropy alloys. Frontiers Mater., 5:42, 2018 . 
[13] H. Song, F. Tian, Q.-M. Hu, L. Vitos, Y. Wang, J. Shen, and N. Chen. Local lattice distortion in high-entropy alloys. Phys. Rev. Mater., 1(2):023404, 2017.

[14] B. Feng and M. Widom. Elastic stability and lattice distortion of refractory high entropy alloys. Mater. Chem. Phys., 210:309-314, 2018.

[15] Y.Y. Zhao and T.G. Nieh. Correlation between lattice distortion and friction stress in Ni-based equiatomic alloys. Intermetallics, 86:45-50, 2017.

[16] H. Chen, A. Kauffmann, S. Laube, I.-C. Choi, R. Schwaiger, Y. Huang, K. Lichtenberg, F. Müller, B. Gorr, H.-J. Christ, and M. Heilmaier. Contribution of lattice distortion to solid solution strengthening in a series of refractory high entropy alloys. Metall. Mater. Trans. A, 49(3):772-781, 2018.

[17] C. Varvenne, A. Luque, and W.A. Curtin. Theory of strengthening in FCC high entropy alloys. Acta Mater., 118:164-176, 2016.

[18] W.G. Nöhring and W.A. Curtin. Correlation of microdistortions with misfit volumes in high entropy alloys. Scripta Mater., 168:119-123, 2019.

[19] B. Yin and W.A. Curtin. Origin of high strength in the CoCrFeNiPd high-entropy alloy. Mater. Res. Lett., 8(6):209-215, 2020.

[20] C. Varvenne and W.A. Curtin. Predicting yield strengths of noble metal high entropy alloys. Scripta Mater., 142:92-95, 2018.

[21] B. Yin and W.A. Curtin. First-principles-based prediction of yield strength in the RhIrPdPtNiCu high-entropy alloy. N.JP Comp. Mater., 5(1):1-7, 2019.

[22] B. Yin, S. Yoshida, N. Tsuji, and W.A. Curtin. Yield strength and misfit volumes of NiCoCr and implications for short-range-order. Nat. Comm., 11(1):2507, 2020.

[23] B. Yin, F. Maresca, and W.A. Curtin. Vanadium is an optimal element for strengthening in both FCC and BCC high-entropy alloys. Acta Mater., 188:486-491, 2020.

[24] F. Maresca and W.A. Curtin. Mechanistic origin of high strength in refractory BCC high entropy alloys up to 1900K. Acta Mater., 182:235-249, 2020.

[25] J.-W. Yeh, S.-Y. Chang, Y.-D. Hong, S.-K. Chen, and S.-J. Lin. Anomalous decrease in $\mathrm{X}$-ray diffraction intensities of $\mathrm{Cu}-\mathrm{Ni}-\mathrm{Al}-\mathrm{Co}-\mathrm{Cr}-\mathrm{Fe}-\mathrm{Si}$ alloy systems with multi-principal elements. Mater. Chem. Phys., 103(1):41-46, 2007.

[26] Y. Zou, S. Maiti, W. Steurer, and R. Spolenak. Size-dependent plasticity in an $\mathrm{Nb}_{25} \mathrm{Mo}_{25} \mathrm{Ta}_{25} \mathrm{~W}_{25}$ refractory high-entropy alloy. Acta Mater., 65:85-97, 2014.

[27] H.S. Oh, D. Ma, G.P. Leyson, B. Grabowski, E.S. Park, F. Körmann, and D. Raabe. Lattice distortions in the FeCoNiCrMn high entropy alloy studied by theory and experiment. Entropy, 18(9):321, 2016.

[28] Y. Tong, K. Jin, H. Bei, J.Y.P. Ko, D.C. Pagan, Y. Zhang, and F.X. Zhang. Local lattice distortion in $\mathrm{NiCoCr}, \mathrm{FeCoNiCr}$ and $\mathrm{FeCoNiCrMn}$ concentrated alloys investigated by synchrotron X-ray diffraction. Mater. Design, 155:1-7, 2018.

[29] L.R. Owen, E.J. Pickering, H.Y. Playford, H.J. Stone, M.G. Tucker, and N.G. Jones. An assessment of the lattice strain in the CrMnFeCoNi high-entropy alloy. Acta Mater., 122:11-18, 2017.

[30] L.R. Owen and N.G. Jones. Lattice distortions in high-entropy alloys. J. Mater. Res., 33(19):2954-2969, 2018.

[31] N.L. Okamoto, K. Yuge, K. Tanaka, H. Inui, and E.P. George. Atomic displacement in the CrMnFeCoNi high-entropy alloy-a scaling factor to predict solid solution strengthening. AIP Advances, 6(12):125008, 2016. 
[32] Y.F. Ye, Y.H. Zhang, Q.F. He, Y. Zhuang, S. Wang, S.Q. Shi, A. Hu, J. Fan, and Y. Yang. Atomic-scale distorted lattice in chemically disordered equimolar complex alloys. Acta Mater., 150:182-194, 2018.

[33] T. Nattermann, S. Stepanow, L.-H. Tang, and H. Leschhorn. Dynamics of interface depinning in a disordered medium. J. Phys. II, 2(8):1483-1488, 1992.

[34] A.-L. Barabási and H.E. Stanley. Fractal concepts in surface growth. Cambridge University Press, 1995.

[35] S. Zapperi and M. Zaiser. Depinning of a dislocation: the influence of long-range interactions. Mater. Sci. Eng. A, 309:348-351, 2001.

[36] J.-H. Zhai and M. Zaiser. Properties of dislocation lines in crystals with strong atomicscale disorder. Mater. Sci. Eng. A, 740:285-294, 2019.

[37] A. Lemaître. Stress correlations in glasses. J. Chem. Phys., 149(10):104107, 2018.

[38] A.G. Khachaturyan. Theory of structural transformations in solids. John Wiley and Sons Inc., 1983.

[39] T. Mura. Micromechanics of defects in solids. Martinus Nijhoff, 1987.

[40] K. Huang. X-ray reflexions from dilute solid solutions. Proc. R. Soc. Lond. Ser A, 190(1020):102-117, 1947.

[41] R.I. Barabash, J.S. Chung, and M.F. Thorpe. Lattice and continuum theories of Huang scattering. J. Phys. Cond. Mat., 11(15):3075, 1999.

[42] M.F. Thorpe, J.S. Chung, and Y. Cai. Diffraction from random alloys. Phys. Rev. B, 43(10):8282, 1991.

[43] E. Antillon, C. Woodward, S.I. Rao, B. Akdim, and T.A. Parthasarathy. Chemical short range order strengthening in a model FCC high entropy alloy. Acta Mater., 190:29-42, 2020 .

[44] C. Varvenne, A. Luque, W.G. Nöhring, and W.A. Curtin. Average-atom interatomic potential for random alloys. Phys. Rev. B, 93(10):104201, 2016.

[45] J.D. Eshelby. The determination of the elastic field of an ellipsoidal inclusion, and related problems. Proc. Roy. Soc. Lond. Ser-A., 241(1226):376-396, 1957.

[46] A.F. Bower. Applied mechanics of solids. CRC Press, 2010.

[47] P. Billingsley. Probability and measure. John Wiley and Sons Inc., 2008.

[48] J.P. Hirth and J. Lothe. Theory of Dislocations. McGraw-Hill, New York, 1968.

[49] D.M. Barnett. The precise evaluation of derivatives of the anisotropic elastic Green's functions. Phys. Stat. Sol. (b), 49(2):741-748, 1972.

[50] X.-Y. Liu and J.B. Adams. Grain-boundary segregation in Al-10\% Mg alloys at hot working temperatures. Acta Mater., 46(10):3467-3476, 1998.

[51] P.-A. Geslin, A. Rida, and D. Rodney. Microelasticity model of random alloys. Part II: displacement and stress correlations. submitted to J. Mech. Phys. Solids, 2020.

[52] S. Plimpton. Fast parallel algorithms for short-range molecular dynamics (http://lammps.sandia.gov). J. Comp. Phys., 117(1):1-19, 1995.

[53] R.O. Scattergood and D.J. Bacon. The Orowan mechanism in anisotropic crystals. Phil. Mag., 31(1):179-198, 1975.

[54] R.O. Scattergood and D.J. Bacon. The strengthening effect of voids. Acta Metall., 30(8):1665-1677, 1982. 
[55] R.M. Elder, W.D. Mattson, and T.W. Sirk. Origins of error in the localized virial stress. Chem. Phys. Lett., 731:136580, 2019.

[56] P.S. Branicio and D.J. Srolovitz. Local stress calculation in simulations of multicomponent systems. J. Comp. Phys., 228(22):8467-8479, 2009.

[57] N.C. Admal and E.B. Tadmor. A unified interpretation of stress in molecular systems. J. Elasticity, 100(1-2):63-143, 2010.

[58] E. Betti. Teoria della elasticita. Il Nuovo Cimento (1869-1876), 7(1):158-180, 1872.

[59] S. Nag and W.A. Curtin. Effect of solute-solute interactions on strengthening of random alloys from dilute to high entropy alloys. Acta Mater., 200:659-673, 2020. 